\title{
A Survey of the Floral Volatile Profiles of Daylily Species and Hybrids
}

\author{
Shea A. Keene, Timothy S. Johnson, Cindy L. Sigler, Terah N. Kalk, Paul Genho, and \\ Thomas A. Colquhoun \\ Environmental Horticulture Department, Plant Innovation Center, Institute of Food and Agricultural \\ Sciences, University of Florida, 2550 Hull Road, Room 1549, P.O. Box 110670, Gainesville, FL 32611
}

\begin{abstract}
Additional INDEX words. fragrance, gas chromatography-mass spectrometry, Hemerocallis, hybridization, monoterpene hydrocarbons, sesquiterpenes, terpenoid derivatives

ABstract. For the past century, daylily (Hemerocallis) hybridization focused almost exclusively on enhancing the diversity of flower forms, colors, and color patterns. This focus on the visual characteristics of daylilies resulted in the development of thousands of hybrids that come in a fantastic array of colors and color patterns. However, these modern daylilies exhibit little to none of the floral fragrance possessed by some of the daylily progenitor species. Because little work has been done on daylily floral volatiles, the objective of this research was to evaluate the floral volatile profiles of a variety of daylily species and hybrids and assess the state of fragrance among modern hybrids. Using a field collection system and gas chromatography-mass spectrometry (GS-MS), this study evaluated the fragrance profiles of 147 daylily genotypes. Eighteen volatile organic compounds, primarily terpenoids, were identified and their variations among the genotypes were investigated. Results suggest that fragrance could be a trait pursued in a breeding program to enhance the sensory phenotypes of new daylily cultivars.
\end{abstract}

Daylilies (Hemerocallis) are monocotyledonous herbaceous perennial plants in the Asphodelaceae family. The genus and common name reference the blooming habit of daylilies: individual daylily flowers reach anthesis and are open for only $1 \mathrm{~d}$. Daylilies are native to subtropical and temperate Asia, arising mainly from China, Korea, and Japan (RodriguezEnriquez and Grant-Downton, 2013). Approximately 20 species are known, the colorations of which are limited to yellow, orange, and fulvous red (Gulia et al., 2009). Daylily hybridization began in earnest in the early 20th century, mostly by amateur breeders that focused on increasing the diversity of flower colors, color patterns, and flower forms (Gulia et al., 2009). Today daylilies come in a dazzling array of colors, patterns, shapes, and sizes, and many of these hybrids bear little or no resemblance to the species to which they are distantly related. Indeed, species are very rarely used in modern daylily breeding; rather, both existing cultivars and unregistered hybrids are used to breed the vast majority of new daylily cultivars (Zhao et al., 2017). The American Daylily Society is the official daylily registrar, and maintains an online database of more than 90,000 registered cultivars (American Daylily Society, 2019). Over the years, the focus on flower color, pattern, and form in daylily breeding resulted in at least one unfortunate, unintended consequence: the loss of fragrance. Although a number of the

Received for publication 20 Sept. 2019. Accepted for publication 10 Dec. 2019. Published online 3 February 2020.

We thank the National Horticulture Foundation, American Floral Endowment, and USDA-ARS Floriculture Nursery Research Initiative for funding this work. We thank Jeff and Elizabeth Salter of Rollingwood Gardens, Jamie and Dianna Gossard of Heavenly Gardens, and Paul Genho for allowing their daylily collections to be part of this research. We also thank Sheldon Nelson, Rick Jellen, and Melissa Fogarty from Brigham Young University for helping obtain materials for the field work in Utah.

T.A.C. is the corresponding author. E-mail: ucntcme1@ufl.edu.

This is an open access article distributed under the CC BY-NC-ND license (https://creativecommons.org/licenses/by-nc-nd/4.0/). daylily species have noticeable, distinct fragrances, those fragrances are thought to be greatly reduced or largely absent in modern hybrids (Jiao et al., 2016). Loss of fragrance is not a phenomenon unique to daylilies. In many types of commercially grown flowers, such as roses (Rosa $\mathrm{sp}$.) and carnations (Dianthus sp.), the selection for traits like postharvest longevity and visual characteristics of the flower, and the failure to select for fragrance traits, has led to a loss of scent (Borda et al., 2011; Dudareva and Pichersky, 2000; Kishimoto et al., 2011). As daylily breeders seek to create novel hybrids that stand out from the crowded field of existing cultivars, they are turning their attention to the long-ignored trait of fragrance.

Floral fragrance is composed of mixtures of volatile organic compounds (VOCs or volatiles) that are mostly lipophilic liquids with high vapor pressures at ambient temperatures (Pichersky et al., 2006). Floral volatiles typically fit in the families of terpenoids, phenylpropanoids, or benzenoids, as well as derivatives of amino acids and fatty acids (Dudareva et al., 2013). Floral volatiles serve a number of biological functions, including attracting pollinators, mediating plantplant interactions, and acting as defense compounds to protect the plant during certain abiotic stresses (Dudareva et al., 2006). Floral volatiles also serve as sensory attractants to humans. In a study on consumer preference for the essential features of a flower product, researchers identified fragrance as one of the most important drivers of consumer liking (Levin et al., 2012). A lack of fragrance negatively affected overall consumer interest the most out of all the flower characteristics evaluated, indicating that consumers prefer fragrant flowers.

Only two studies have been conducted on daylily fragrance, both in China (Jiao et al., 2016; Lin et al., 2003). Lin et al. (2003) subjected Hemerocallis flava flowers to simultaneous distillation-solvent extraction. They analyzed the resulting essential oil using GC-MS and identified 51 compounds. However, depending on the extraction process, the aroma of an essential oil can differ from the aroma experienced by a 
person smelling the flower from which the oil came (Tholl et al., 2006). Using headspace solid-phase microextraction and GCMS, Jiao et al. (2016) evaluated the volatile emissions of seven daylily species, one botanical variety, and 38 cultivars. They identified 37 volatiles, most of which were terpenoids. These studies provide a point of reference for further investigation of daylily scent, but ultimately only reflect the volatiles emitted by a tiny fraction of the expansive modern daylily germplasm.

The objectives of this research were to evaluate the volatile profiles of a larger number of daylily hybrids and some species grown in three locations across the United States, to identify volatile compounds in those profiles, assess the variation of volatile emissions among different daylilies, and finally to determine which genotypes may be genetic resources of volatile compounds. Hybridizers looking to pursue aroma traits in their breeding programs could use this information to select for daylily hybrids with enhanced scent, and potentially develop unique cultivars with exceptional fragrance that appeal to more horticultural consumers.

\section{Materials and Methods}

Study Sites AND Plant Material. Volatiles were sampled in situ from three collections of daylilies during the bloom season. In general, daylilies bloom over the summer, although the peak blooming period varies according to location. The first group, owned and managed by small-scale commercial breeders, was located in Archer, FL (lat. 29 $36^{\prime} 57.6^{\prime \prime} \mathrm{N}$, long. 82 $31^{\prime} 26.4^{\prime \prime} \mathrm{W}$; approximate elevation $25.0 \mathrm{~m}$ ). Volatiles were collected from 24 registered cultivars, 37 "seedlings" (a term used in the daylily community to refer to unregistered hybrid offspring), and three species $(n=64)$. Volatile sampling occurred between 13 May 2016 and 11 July $2016\left(32.8^{\circ} \mathrm{C}\right.$ average high temperature and $70 \%$ average relative humidity). The second group, owned and managed by a germplasm collector and large-scale commercial breeder, was located in Galloway, $\mathrm{OH}$ (lat. $39^{\circ} 58^{\prime} 12.0^{\prime \prime} \mathrm{N}$, long. $83^{\circ} 12^{\prime} 36.0^{\prime \prime} \mathrm{W}$; approximate elevation $275 \mathrm{~m})$. Volatiles were collected from 25 cultivars, five seedlings, two species, and one botanical variety $(n=33)$. Sampling occurred between 10 July 2017 and 12 July 2017 (28.7 ${ }^{\circ} \mathrm{C}$ average high temperature and $87 \%$ average relative humidity). The third group, owned and managed by a small-scale hobbyist breeder, was located in Payson, UT (lat. $40^{\circ} 01^{\prime} 48.0^{\prime \prime} \mathrm{N}$, long. $111^{\circ} 46^{\prime} 12.0^{\prime \prime} \mathrm{W}$; approximate elevation $\left.1400 \mathrm{~m}\right)$. Volatiles were collected from 48 cultivars and two seedlings $(n=50)$. Sampling occurred between 14 July 2017 and 19 July 2017 $\left(35.6^{\circ} \mathrm{C}\right.$ average high temperature and $42 \%$ average relative humidity). The presence of two fully open flowers was the main selection criterion for volatile sampling. Beyond that, daylilies of as many colors, color patterns, and flower forms as possible were selected at random from the collections. The supplemental spreadsheet file provides the name, location, ploidy (if known), and registration status of each daylily evaluated (Supplemental Table 1).

Volatile Collection. Volatiles were sampled via headspace sorption between 1000 and $1400 \mathrm{HR}$. This time period was chosen in part because of the blooming habit of daylilies: between 1000 and $1400 \mathrm{HR}$, the daylily flowers are fully open and have not begun to senesce. Moreover, as these collections were done in the field during the summer, temperature and the chance of precipitation were taken into consideration. Volatiles were collected in duplicate from each genotype. Flowers were inserted into a $35.5 \times 50.8-\mathrm{cm}$ nylon resin cooking bag (MasterPack; Reynolds Consumer Products, Lake Forest, IL) and the bag was gathered around the scape beneath the flower and cinched with a twist tie over a cotton plug (Stewart-Jones and Poppy, 2006). Plastic bags have been used extensively in previous volatile research (Huber et al., 2005; Kessler and Baldwin, 2007; Raguso et al., 2003; Stewart-Jones and Poppy, 2006 and references therein). A glass column containing $\approx 50$ mg porous polymer adsorbent (HaySep Q 80-100; Hayes Separations, Bandera, TX) was inserted into a slit at the top of the bag above the flower and secured with a twist tie. The glass column was fastened to a wooden stake to prevent the collection apparatus from collapsing on the flower. A schematic diagram of the collection system is shown in Fig. 1. Following an hour of volatile enrichment, a single-setting vacuum pump (Barnant Co., Barrington, IL) was used to pull the air out of the bag through the adsorbent trap for 3 minutes. On each collection date, volatiles were sampled from empty nylon resin bags to account for background contaminants. Volatiles were eluted from the adsorbent polymer within $12 \mathrm{~h}$ with $150 \mu \mathrm{L}$ of methylene chloride (CAS 75-09-2) spiked with $25 \mathrm{ng} \cdot \mu \mathrm{L}^{-1}$ of nonyl acetate (CAS 143-13-5) as an elution standard (Thermo Fisher Scientific, Waltham, MA). Samples were stored at $-80^{\circ} \mathrm{C}$ until analysis by GC-MS.

VolATILE ANALYSIS. Volatile samples were analyzed on a gas chromatograph (7890A; Agilent Technologies, Santa Clara, CA) fitted with a DB-5 column (5\% phenyl, 95\% dimethylpolysiloxane, $29 \mathrm{~m}$ length $\times 0.25 \mathrm{~mm}$ internal diameter $\times 1 \mu \mathrm{m}$ film thickness) and coupled to a mass spectrometer (5977A; Agilent Technologies). Helium was used as a carrier gas with a fixed pressure of $93.5 \mathrm{kPa}$. Samples were injected at a volume of $2 \mu \mathrm{L}$ and then split at a ratio of $20: 1$. The injection temperature was $220^{\circ} \mathrm{C}$. The oven temperature was programmed from $40^{\circ} \mathrm{C}$ with a 0.5 -min hold to $250{ }^{\circ} \mathrm{C}$ with a 4 -min hold, at $5{ }^{\circ} \mathrm{C}$ per minute. Compounds were tentatively identified by comparing their mass spectra to the 2011 National Institute of Standards and Technology mass spectral library. Volatile identification was achieved by comparing the retention times and mass spectra of peaks in the samples to those of authentic standards (Sigma-Aldrich, St. Louis, MO, and Tokyo Chemical Industry, Portland, OR). Analysis of volatile data were performed using MassHunter Qualitative (version B.06.00; Agilent Technologies) and Quantitative (version B.05.02; Agilent Technologies) software programs. Relative volatile emission was calculated based on individual peak area relative to the peak area of the elution standard within each sample. Calibration curves for authentic standards were run in duplicate on the GC-MS under the same conditions described above.

Statistical ANALYSIS. For all volatile emission replicates, means and standard errors were calculated using JMP Pro (version 14.1.0; SAS Institute, Cary, NC). Because of the size of this study's dataset, as well as the variability of daylily volatile composition and emissions, two complementary multivariate methods were used to analyze the data. Principal component analysis (PCA) is an exploratory, multivariate technique that reduces the dimensionality of a large dataset and provides insight into the associations among variables. The application of PCA to volatile data is particularly valuable. It can reveal patterns in the data that might otherwise be obscured by highly correlated volatiles arising from the same biochemical pathway, and it also helps identify volatiles that are most differentiating among genotypes (Granato et al., 2017; Majetic 


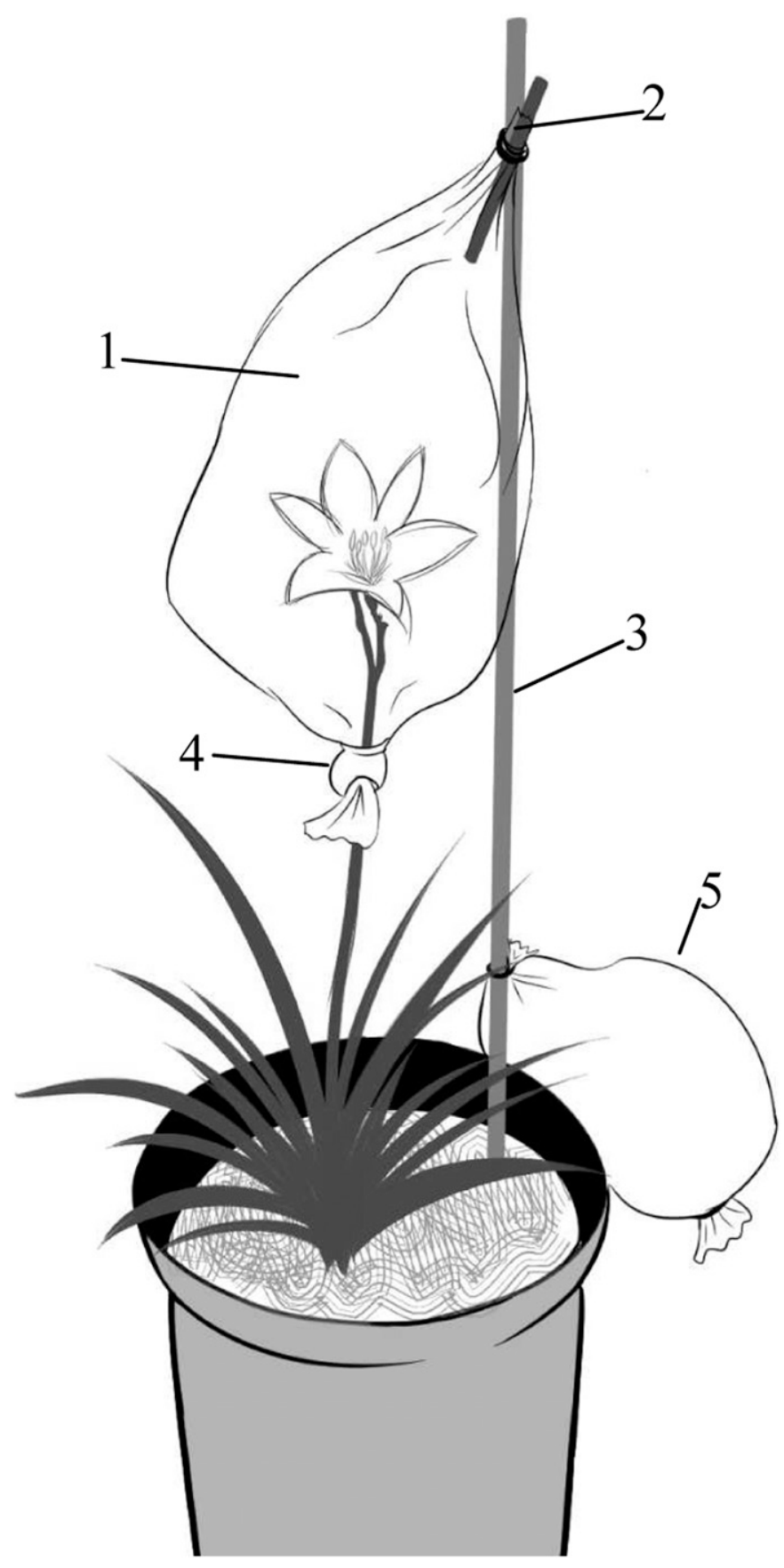

Fig. 1. Schematic diagram of the collection system used in this study of daylily volatiles showing the (1) nylon resin oven bag used as collection chamber, (2) glass column containing adsorbent polymer, (3) wooden stake to support the collection apparatus, (4) cinching of the bag over a cotton plug to cushion the scape, and (5) empty bag from which volatiles were sampled to account for background contaminants.

et al., 2007). Hierarchical cluster analysis (HCA) is an exploratory, multivariate technique that visually represents relationships in the data, typically through dendrograms (Granato et al., 2017). PCA in JMP Pro was performed on the dataset from each study location. For these analyses, a PCA was run that excluded volatiles detected in fewer than $20 \%$ of the daylilies evaluated. Because the excluded volatiles were completely absent in many samples, their inclusion could introduce bias to the analysis (Majetic et al., 2007). To visualize how daylily genotypes group together based on their volatile emissions, and to visualize the differences in the amount of volatile compounds emitted among the daylily genotypes, two-way Ward's HCA was performed on the dataset, excluding volatiles detected in fewer than $20 \%$ of the daylilies evaluated, from each study location using the default settings in JMP Pro.

\section{Results}

VOLATILE COMPOSITION OF DAYLILY FRAGRANCE. The volatile profiles of five daylily species, one botanical variety, 97 registered cultivars, and 44 seedlings were evaluated, and 18 VOCs were identified (Table 1). The number of volatile compounds emitted by individual daylilies ranged from 4 to 16; on average, daylilies emitted 10 volatiles. Most of the 18 volatiles identified in this study were terpenoid compounds, including monoterpene hydrocarbons, monoterpene alcohols, sesquiterpenes, and terpenoid derivatives. Other chemical classes of identified volatiles include benzenoid, aldehyde, alcohol, ketol, fatty-acid-derived, phenylpropanoid-related, and nitrogen-containing compounds. Except for the irregular terpene 6methyl-5-hepten-2-one, all other volatiles were emitted by daylilies in each study location (Fig. 2). The most abundant and prevalent compounds were the monoterpene hydrocarbons (Z)- $\beta$-ocimene and (E)- $\beta$-ocimene (Fig. $2 \mathrm{~A}$ ). These compounds appeared in the volatile profile of every daylily sampled, and together accounted for an average of almost $83 \%$ of the total amount of volatiles emitted. The volatiles differed in their amounts of relative emission among the genotypes. Some volatiles were emitted over a small range of concentrations, whereas others were emitted over a large range (Table 1). For example, the maximum amount of phenylethyl alcohol emitted by the seedling "1287-13" was five times more than the minimum amount emitted by 'Spacecoast Blue Eyed Majesty', whereas the maximum amount of $(E)$-2-methyl-2-butenal emitted by 'Bright Blaze of Magic' was 2970 times greater than the minimum amount emitted by 'Wind Rider'. Fourteen volatiles were emitted, on average, at amounts of less than 1.20 $\mu \mathrm{g} /$ flower (Fig. 2B). Only four volatiles were considered "high abundance," with average emission amounts of more than 1.40 $\mu \mathrm{g} /$ flower (Fig. 2A). The supplemental Excel file contains the full dataset of means and standard errors of volatile compound replicates for each daylily evaluated (Supplemental Table 1).

TOTAL VOLATILE EMISSIONS VARY AMONG DAYLILY GENOTYPES. The total amount of volatiles emitted by individual daylilies ranged from 0.92 to $159.54 \mu \mathrm{g}$ (Table 2). The five highest and lowest producers of floral volatiles for each study site are also shown in Table 2. In Florida, the most fragrant daylily (in terms of total volatile emissions) was the species Hemerocallis thunbergii. This species emitted almost 80 times the total VOCs as 'Rim of Fire', the daylily with the lowest total emissions. The most fragrant hybrid genotype, 'Spacecoast Blue Eyed Majesty', emitted more than 60 times more VOCs than 'Rim of Fire'. More than $80 \%$ of the genotypes evaluated released less than half the amount of volatiles as $H$. thunbergii; however, almost $17 \%$ of the genotypes emitted more than half, including 'Spacecoast Blue Eyed Majesty', 'Bridge of Dreams', and 'Jalapeno Crunch'. Although average total VOC emissions were slightly more than 54 $\mu \mathrm{g} /$ flower, these hybrids released more than $100 \mu \mathrm{g} /$ flower.

In Ohio, the most fragrant daylily was again a species, this time Hemerocallis citrina. The amount of fragrance released by $H$. citrina was 165 times greater than the amount released by the 
Table 1. Summary statistics of volatile compounds identified in daylily study population $(\mathrm{N}=147)$. Volatiles are listed in ascending order of gas chromatography-mass spectrometry retention time.

\begin{tabular}{|c|c|c|c|c|c|c|c|c|c|}
\hline \multirow[b]{2}{*}{ Compound } & \multirow[b]{2}{*}{ CAS $^{z}$} & \multirow[b]{2}{*}{ No. ${ }^{y}$} & \multirow[b]{2}{*}{$\%^{\mathrm{y}}$} & \multicolumn{4}{|c|}{ Volatile emission $(\mu \mathrm{g} /$ flower $)$} & \multirow[b]{2}{*}{ Fold diff. ${ }^{\mathrm{x}}$} & \multirow{2}{*}{$\begin{array}{l}\text { Daylily emitting } \\
\text { maximum }\end{array}$} \\
\hline & & & & Minimum & Mean \pm SE & Median & Maximum & & \\
\hline Acetoin & $513-86-0$ & 98 & 66 & 0.10 & $0.39 \pm 0.03$ & 0.31 & 2.39 & 24 & Bright Blaze of Magic \\
\hline 2-Methyl-1-butanol & $137-32-6$ & 26 & 17 & 0.07 & $0.26 \pm 0.03$ & 0.19 & 0.69 & 9 & Celtic Witch \\
\hline (E)-2-Methyl-2-butenal & $497-03-0$ & 111 & 75 & 0.001 & $0.35 \pm 0.04$ & 0.21 & 2.31 & 2970 & Bright Blaze of Magic \\
\hline 3-Methyl-2-butenal & $107-86-8$ & 123 & 83 & 0.07 & $1.88 \pm 0.19$ & 1.24 & 11.24 & 161 & Cheddar Explosion \\
\hline Hexanal & $66-25-1$ & 49 & 33 & 0.02 & $0.22 \pm 0.03$ & 0.13 & 0.70 & 32 & 2016 No Name (2) \\
\hline Benzaldehyde & $100-52-7$ & 34 & 23 & 0.05 & $0.15 \pm 0.02$ & 0.11 & 0.46 & 9 & Cheddar Explosion \\
\hline 6-Methyl-5-hepten-2-one & $110-93-0$ & 6 & 4 & 0.01 & $0.03 \pm 0.01$ & 0.03 & 0.05 & 7 & Jalapeno Crunch \\
\hline$\beta$-Myrcene & $123-35-3$ & 136 & 92 & 0.06 & $0.77 \pm 0.04$ & 0.64 & 3.00 & 47 & Blue Vibrations \\
\hline 2-Ethyl-1-hexanol & $104-76-7$ & 139 & 94 & 0.05 & $0.24 \pm 0.01$ & 0.21 & 0.67 & 14 & Wind Rider \\
\hline (Z)- $\beta$-Ocimene & $3779-61-1$ & 147 & 100 & 0.33 & $9.22 \pm 0.59$ & 8.00 & 40.52 & 122 & H. citrina \\
\hline (E)- $\beta$-Ocimene & $3338-55-4$ & 147 & 100 & 0.05 & $33.67 \pm 1.81$ & 32.47 & 94.33 & 1725 & H. thunbergii \\
\hline$\beta$-Linalool & $78-70-6$ & 92 & 62 & 0.02 & $0.30 \pm 0.03$ & 0.21 & 1.60 & 94 & H. thunbergii \\
\hline Phenylethyl alcohol & $60-12-8$ & 102 & 69 & 0.12 & $0.33 \pm 0.01$ & 0.28 & 0.66 & 5 & $1287-13$ \\
\hline allo-Ocimene & $673-84-7$ & 115 & 78 & 0.01 & $0.04 \pm 0.003$ & 0.03 & 0.18 & 26 & H. citrina \\
\hline Indole & $120-72-9$ & 61 & 41 & 0.05 & $0.49 \pm 0.07$ & 0.25 & 2.74 & 53 & Oh Great One \\
\hline$\beta$-Caryophyllene & $87-44-5$ & 20 & 13 & 0.03 & $0.22 \pm 0.03$ & 0.17 & 0.50 & 20 & Red Double 2016 \\
\hline$\alpha$-Farnesene & $502-61-4$ & 97 & 65 & 0.25 & $4.27 \pm 0.58$ & 2.31 & 41.35 & 165 & H. thunbergii \\
\hline (E)-Nerolidol & $40716-66-3$ & 71 & 48 & 0.04 & $0.34 \pm 0.05$ & 0.20 & 1.90 & 51 & Wind Rider \\
\hline
\end{tabular}

${ }^{\mathrm{z}} \mathrm{CAS}=$ Chemical Abstract Service, the unique numerical identifier of a substance.

${ }^{\mathrm{y}}$ Number of daylilies that emitted a given volatile; \% denotes the percentage of the study population that emitted a given volatile.

${ }^{\mathrm{x}}$ Fold difference, rounded to the nearest whole number, is calculated by dividing the unrounded maximum by the unrounded minimum value for each compound.

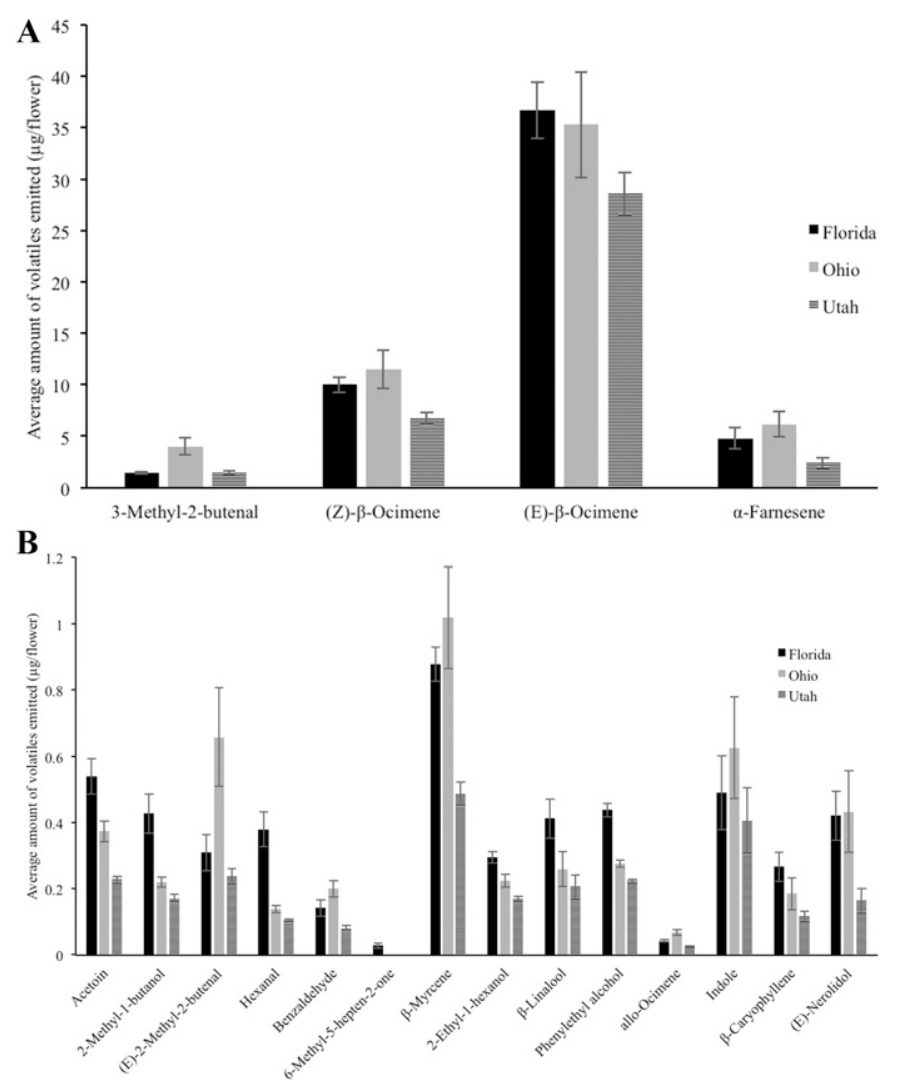

Fig. 2. Average amount of the four "high-abundance" volatile compounds, accumulating at $>1.4 \mu \mathrm{g} /$ flower (A), and of the 14 "low abundance" compounds, accumulating at $<1.2 \mu \mathrm{g}$ /flower (B), emitted by daylilies in each study location. Standard error bars are shown for each volatile mean. least-scented genotype, the species clone Hemerocallis fulva 'Korean' (Table 2). The hybrid with the highest total VOC emission, 'Out of Balance', released $\approx 135$ times more fragrance than H. fulva 'Korean', and 100 times more fragrance than the least-fragrant hybrid, an unnamed seedling. Approximately $36 \%$ of the hybrids emitted more than half the amount emitted by $H$. citrina. These genotypes included 'Out of Balance', 'Popcorn at the Movies', 'Blue Vibrations', 'Spacecoast Devil's Eye', 'Mystical Elf', and 'Blackwater Captain Jack'. Although average total VOC emissions were $\approx 56$ $\mu \mathrm{g} /$ flower, these genotypes all emitted more than $100 \mu \mathrm{g} /$ flower.

In Utah, total volatile emissions were lower compared with the other two study locations. Consequently, the range of total volatile emissions was smaller, with the most-scented genotype, 'Cranberry Daiquiri', releasing 56 times more volatiles than the least-scented genotype, 'Cheers for Now' (Table 2).

PCA AND WARD'S HCA IDENTIFY A SUBSET OF DAYLILIES WITH EXCEPTIONAL FRAGRANCE PROFILES. Because of the differences among the three study collections in terms of climate, breeder priorities, management practices such as fertilization and irrigation regimens, soil type, and other factors, the dataset from each daylily collection was analyzed individually.

FLORIDA PCA. Together, the first two principal components (PC) explained $50 \%$ of the total variance in the volatile data of the Florida collection (Fig. 3, Supplemental Fig. 1). The loading coefficients of the volatiles for the first two PCs are shown in Supplemental Table 2. These coefficients indicate how much a compound affects a given PC. A compound that explains more variance is given a higher weight or loading, whereas a compound that explains little or no variance will have a loading value closer to zero. A high degree of overlap was observed for most of the daylilies in Florida, suggesting that many of the daylilies have similar volatile profiles; however, several 
Table 2. For each study location, total volatile emission summary statistics are provided along with the five daylilies emitting the highest, and the five daylilies emitting the lowest, total amount of volatiles.

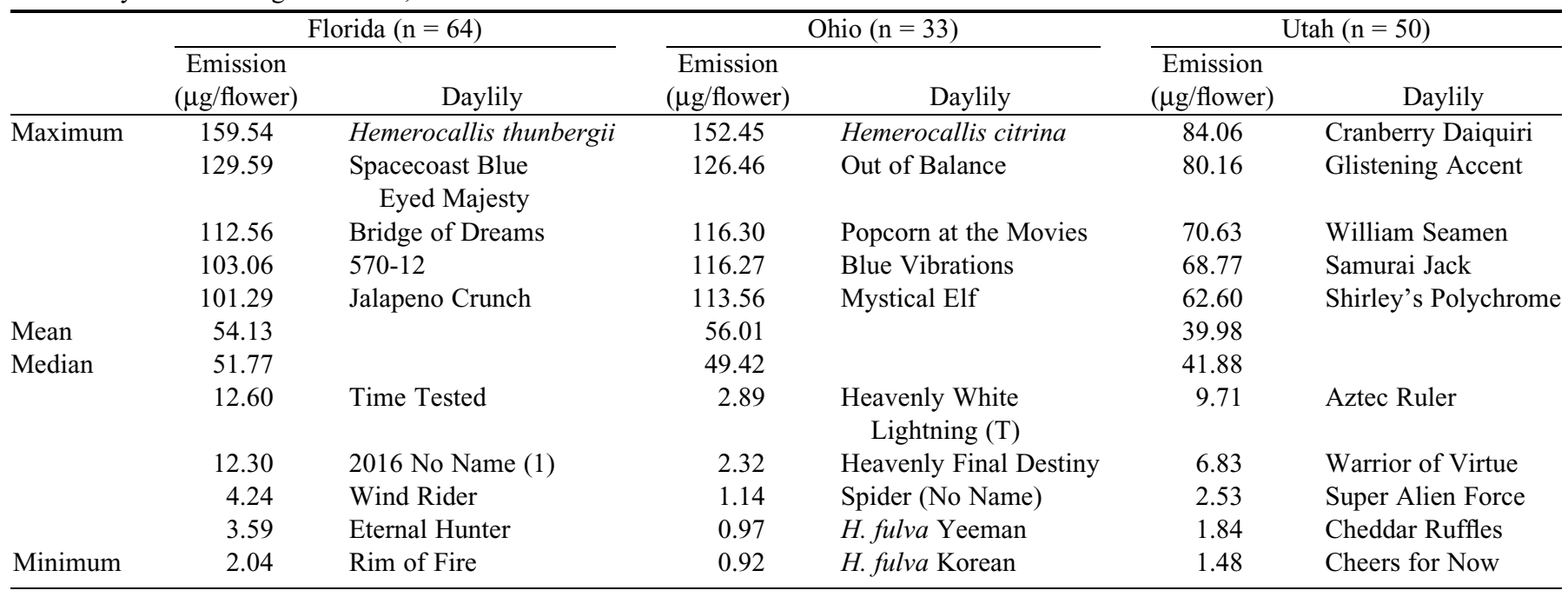

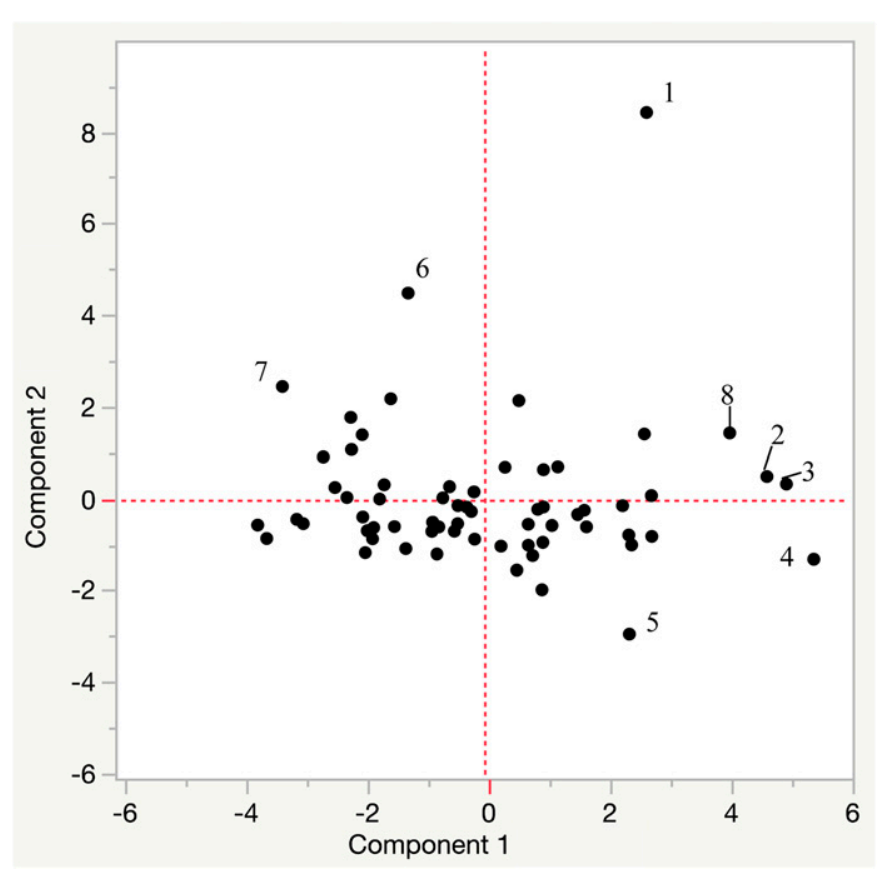

Fig. 3. Score plot of the first two principal components based on 14 volatile compounds detected in the floral scent of daylilies from Florida $(n=64)$. Components 1 and 2 explained $32.5 \%$ and $17.5 \%$, respectively, of the total observed variation. Several daylilies differentiated from the large central grouping, including (1) Hemerocallis thunbergii, (2) 'Spacecoast Blue Eyed Majesty', (3) 'Jalapeno Crunch', (4) 'Bridge of Dreams', (5) 'Bright Blaze of Magic', (6) 'Circus Truffle', (7) 'Wind Rider', and (8) "570-12," an unregistered seedling.

daylilies did not cluster with the rest (Fig. 3). The species $H$. thunbergii is the most isolated point on the score plot. This species emitted the most $\alpha$-farnesene and $\beta$-linalool of any daylily evaluated. These compounds loaded strongly and positively onto PC 2. Other daylilies distinguished on the score plot included 'Jalapeno Crunch' and 'Spacecoast Blue Eyed Majesty', and the seedling " $570-12$." These daylilies were characterized by high emissions of $\beta$-myrcene, allo-ocimene, and
(E)- $\beta$-ocimene; moreover, these daylilies, along with $\mathrm{H}$. thunbergii, had the highest total emissions of the daylilies evaluated in Florida (Table 2). 'Bridge of Dreams' released high levels of these compounds as well, but was further characterized by its elevated emissions of 3-methyl-2-butenal and (E)-2-methyl-2butenal. Three other daylilies separated from the large central cluster: 'Winder Rider' emitted the most $(E)$-nerolidol of any daylily evaluated, 'Circus Truffle' emitted the most indole of any daylily in Florida, and 'Bright Blaze of Magic' emitted more acetoin and more (E)-2-methyl-2-butenal than any daylily evaluated.

Florida HCA. The Florida daylilies clustered into seven groups (Fig. 4). The large Cluster I is characterized by medium emissions of acetoin, (E)-2-methyl-2-butenal, and 3-methyl-2butenal; higher emissions of phenylethyl alcohol; and medium emissions of the monoterpene hydrocarbons. None of these daylilies separated from the central grouping on the PCA score plot (Fig. 3). Cluster II contained four daylilies and was characterized by very high emissions of the monoterpene hydrocarbons: $\beta$-myrcene, $(\mathrm{Z})-\beta$-ocimene, allo-ocimene, and $(E)-\beta$-ocimene. These daylilies clearly separated from the central cluster on the PCA score plot. The third cluster, containing only the daylily 'Bright Blaze of Magic', is characterized by extremely high emissions of acetoin and $(E)-2-$ methyl-2-butenal. This daylily also separated from the others on the PCA score plot. Cluster IV is characterized by high emissions of hexanal and phenylethyl alcohol. Cluster V contains the most genotypes and is defined by low to mid emissions of most volatiles. Cluster VI is characterized by lower emissions of the monoterpene hydrocarbons, as well as medium to high emissions of indole, $\alpha$-farnesene, and $(E)$ nerolidol. Finally, Cluster VII contains a single daylily, $H$. thunbergii. As noted previously, this daylily emitted high amounts of all the terpenoids, the sesquiterpenes, and indole.

Онго PCA. Together, the first two PCs explained $62.8 \%$ of the total variance in the volatile data of the Ohio daylilies (Fig. 5, Supplemental Fig. 2, Supplemental Table 2). Compared with the PCA of daylilies in Florida and Utah, less overlap was seen among the genotypes sampled in Ohio, suggesting greater dissimilarity in their volatile profiles (Fig. 5). The most isolated 


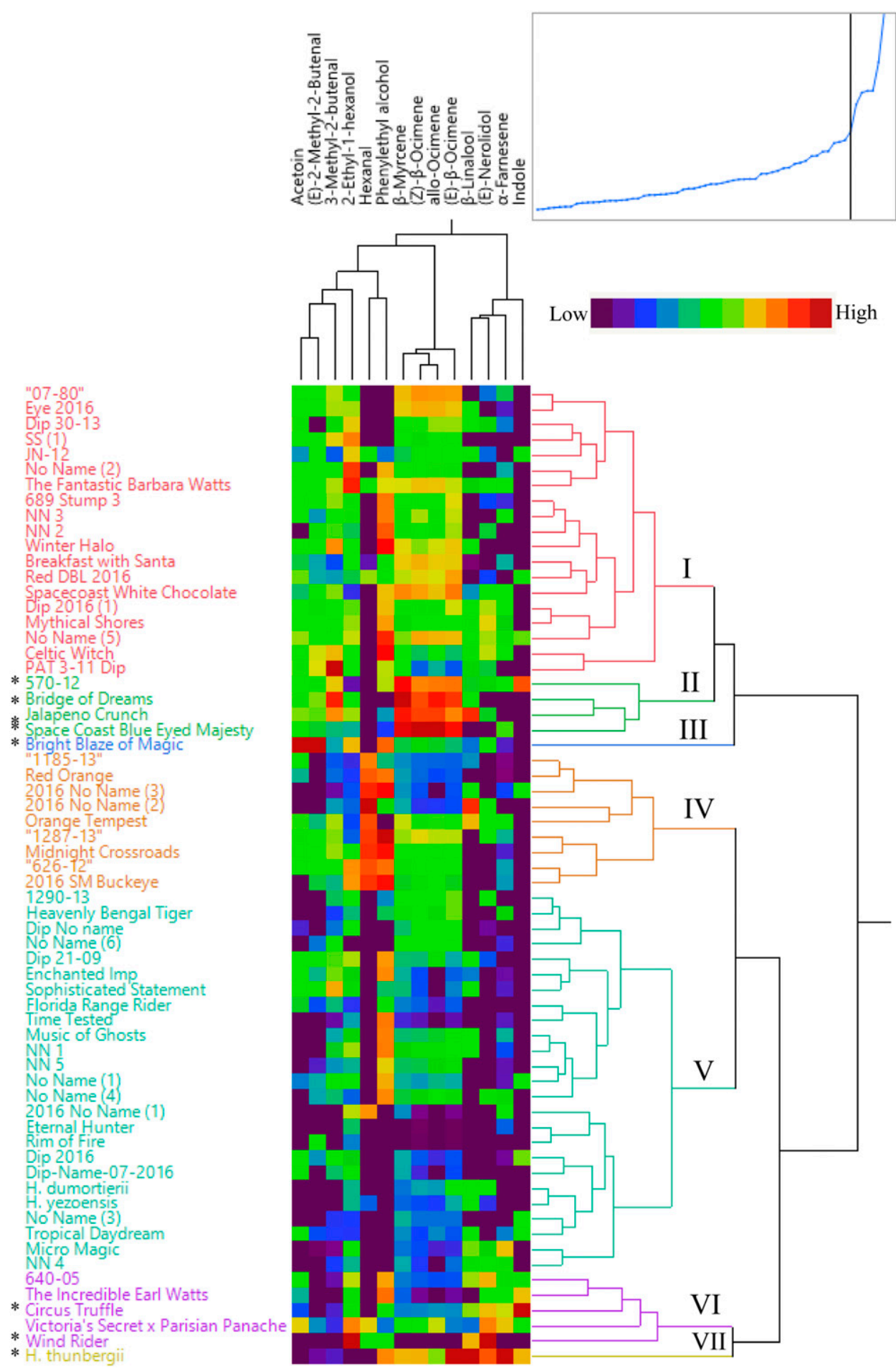

Fig. 4. Two-way cluster analysis of volatile compounds and Florida daylilies $(n=64)$. Daylilies clustered into seven groups, labeled I to VII on the right side of the dendrogram. Daylilies that differentiated on the Florida principal component analysis score plot are marked by an asterisk. Standardization of volatile content is by column mean and SD, with high values represented as red, average as green, and low as violet (spectral scale). (For interpretation of the references to color in this figure legend, the reader is referred to the online version of this article.)

point on the score plot is the species $H$. citrina. As noted previously, this species was the most fragrant Ohio daylily in terms of total volatile emissions. In addition, it had the highest emissions of (Z)- $\beta$-ocimene, (E)- $\beta$-ocimene, and allo-ocimene. Located halfway between $H$. citrina and the PC 2 axis, 'Out of
Balance' also emitted high levels of these terpenoids, as did 'Mystical Elf' and 'Spacecoast Devil's Eye' clustered nearby. Except for the latter cultivar, these daylilies had some of the highest total emissions of the genotypes evaluated in Ohio (Table 2). One other genotype separated from the others in the first quadrant. 'Blue Throated Hummingbird', located almost directly on the PC 2 axis, was characterized by high emissions of hexanal and 2ethyl-1-hexanol, as well as the highest emission of $\alpha$-farnesene of any genotype in Ohio.

In a separate quadrant, the high emitting 'Blue Vibrations' loosely clustered with four yellow cultivars exhibiting double flower forms. These doubles included 'Double Yellow Thunder', 'Double Rays of Sunshine', 'Popcorn at the Movies', and 'Cheddar Explosion'. The first two doubles emitted high amounts of 3-methyl-2-butenal and (E)-2methyl-2-butenal, whereas the latter two doubles and 'Blue Vibrations' all had high emissions of $(E)$ nerolidol, 3-methyl-2-butenal, and (E)-2-methyl-2-butenal. These three compounds were negatively associated with PC 2.

Онго НCA. The Ohio genotypes clustered into four groups (Fig. 6). Cluster I is characterized by high emissions of acetoin, $(E)$-2-methyl2-butenal, and 3-methyl-2-butenal. Several of the daylilies in this group clustered together on the PCA score plot (Fig. 5). Cluster II is less welldefined, but most of the genotypes in this group emitted high levels of phenylethyl alcohol, as well as medium to high amounts of $\alpha$-farnesene. Cluster III, the smallest cluster, contained four genotypes: $H$. citrina, 'Mystical Elf', 'Out of Balance', and 'Spacecoast Devil's Eye'. This cluster is characterized by medium to high emissions of most volatiles, particularly the monoterpene hydrocarbons. These genotypes separated from the main cluster on the PCA score plot. Cluster IV, the largest group, is characterized by low emissions of most volatiles. None of the genotypes in this group separated from the central cluster on the PCA score plot.

UTAн PCA. Together, the first two PCs explained 57.7\% of the total variance in the volatile data of the Utah daylily collection (Fig. 7, Supplemental Fig. 3, Supplemental Table 


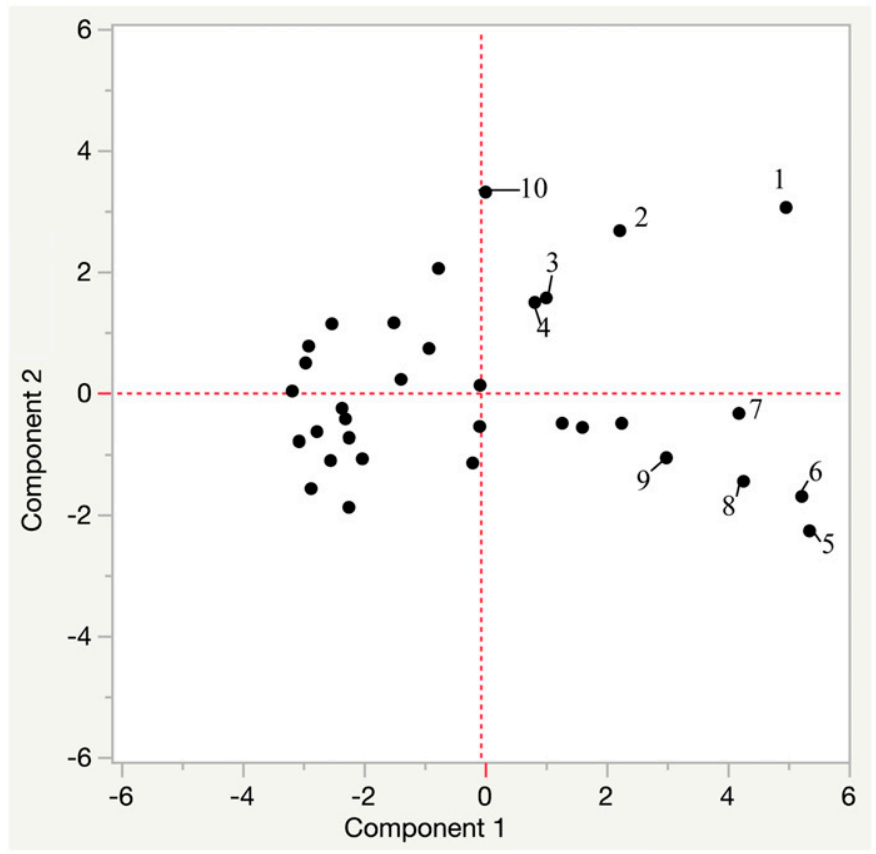

Fig. 5. Score plot of the first two principal components based on 15 volatile compounds detected in the floral scent of daylilies from Ohio $(n=33)$. Components 1 and 2 explained 49.4\% and 13.4\%, respectively, of the total observed variation. Less overlap was seen among these daylilies, including (1) Hemerocallis citrina, (2) 'Out of Balance', (3) 'Mystical Elf', (4) 'Spacecoast Devil's Eye', (5) 'Cheddar Explosion', (6) 'Blue Vibrations', (7) 'Double Yellow Thunder', (8) 'Popcorn at the Movies', (9) 'Double Rays of Sunshine', and (10) 'Blue Throated Hummingbird'.

2). A high degree of overlap was seen among the Utah daylilies, suggesting that the volatile profiles of those in the main grouping shared similarities; however, some cultivars stood slightly apart from the rest on the score plot (Fig. 7). 'Cranberry Daiquiri' had the highest total VOC emissions (Table 2), as well as the highest emissions of $\beta$-myrcene, $(E)$ - $\beta$-ocimene, and allo-ocimene, of any daylily in Utah. Located between 'Cranberry Daiquiri' and the main group on the score plot, 'Glistening Accent' also emitted relatively high levels of these terpenoids. Nearby, 'Samurai Jack' and 'Lover's Lemonade' partially overlapped. These genotypes were characterized by relatively high emissions of benzaldehyde and $(E)$-2-methyl-2butenal. Elsewhere, two cultivars clustered near the PC 2 axis. Although 'Oh Great One' and 'Purple Fantasia' had low total VOC emissions overall, the former cultivar emitted more indole than any daylily evaluated, and the latter cultivar emitted the most $(E)$-nerolidol of any daylily in Utah. Indole and $(E)$-nerolidol loaded positively and with moderate strength onto PC 2.

UTAн HCA. The Utah genotypes clustered into five groups (Fig. 8). Cluster I is characterized by low to medium emissions of acetoin, (E)-2-methyl-2-butenal, 3-methyl-2-butenal, and the monoterpene hydrocarbons. Cluster II has medium to high emissions of these volatiles, as well as medium to high emissions of 2-ethyl-1-hexanol and phenylethyl alcohol. This cluster contains two genotypes, 'William Seamen' and "Shirley's Polychrome", that were among the top five highest emitters of any daylily evaluated in Utah (Table 2). Cluster III contains only three genotypes, all of which separated from the main cluster on the PCA score plot (Fig. 7) and had some of the highest total emissions of the daylilies evaluated in Utah (Table 2). These genotypes are characterized by medium to high emissions of nearly every volatile. Cluster IV contains four genotypes, most of which differentiated on the PCA score plot. These genotypes are characterized by medium emissions of most volatiles, and medium to high emissions of $(E)$-2-methyl-2-butenal, 3-methyl-2butenal, and $(E)$-nerolidol. The final and largest cluster, Cluster $\mathrm{V}$, is defined by low emissions of many of the volatiles, although a subset of the group is characterized by relatively high emissions of phenylethyl alcohol and 2-methyl-1-butanol.

\section{Discussion}

The purpose of this research was to evaluate the volatile profiles of daylily genotypes in three locations across the United States with the goals of identifying important volatile constituents, assessing volatile variation among the genotypes, and identifying daylilies that are genetic resources of aroma compounds. Terpenoids dominated the fragrance profile of every daylily evaluated in this study. Similarly, Jiao et al. (2016) reported that terpenoids represented more than $80 \%$ of the total volatiles released, with $(E)$ - $\beta$-ocimene appearing in the volatile profile of 45 of the 46 daylilies they evaluated. This compound is one of the most common components of floral scent, occurring in more than $70 \%$ of seed plant families (Knudsen et al., 2006). Of the 18 volatiles identified in this study, at least five have not been reported in daylilies previously, including 2-methyl-1-butanol, (E)-2-methyl-2-butenal, 3-methyl-2-butenal, hexanal, and 6-methyl-5-hepten-2-one. The compound 6-methyl-5-hepten-2-one was detected in the fragrance profile of only six daylilies, all grown in Florida. This irregular terpene has a floral fruity aroma and has been documented in more than 45 families of seed plants, including Rosaceae, Malvaceae, and Apiaceae (Knudsen et al., 2006; Widjaja et al., 1996). At least nine volatiles from this study were reported by Jiao et al. (2016), including $\beta$-myrcene, (E)- $\beta$-ocimene, $\beta$-linalool, allo-ocimene, $\beta$-caryophyllene, $\alpha$-farnesene, $(E)$-nerolidol, phenylethyl alcohol, and indole. Lin et al. (2003) also reported phenylethyl alcohol, as well as acetoin, 2-ethyl-1-hexanol, and benzaldehyde. The identification of these compounds in multiple independent studies suggests that these volatiles are indeed part of the fragrance profiles of many daylilies. Along with $(E)$ - $\beta$-ocimene and 6-methyl-5hepten-2-one, the compounds benzaldehyde, $\beta$-myrcene, $\beta$-linalool, $\beta$-caryophyllene, and phenylethyl alcohol are some of the most commonly emitted floral volatiles. Because of their widespread distribution, these compounds are thought to play other important roles aside from attracting pollinators, such as defending against herbivory and improving resistance to abiotic stresses (Knudsen et al., 2006).

An unexpected number of volatiles reported by Jiao et al. (2016) and Lin et al. (2003) in their analysis of 46 genotypes and $H$. flava, respectively, were not detected in the daylilies in this study. Authentic standards of these compounds were run on the GC-MS because they appeared in previous daylily literature; however, subsequent analysis of their mass spectra and retention times determined they were not present in our study population. Of the volatiles listed in Jiao et al. (2016), 3-carene (13466-78-9), $\alpha$-terpinene (99-86-5), $\gamma$-terpinene (99-85-4), terpinolen (586-62-9), and humulene (6753-98-6) were not detected. These volatiles may be unique to the daylilies evaluated by Jiao et al. (2016), or perhaps they were emitted at levels too low to detect with the field collection system used in 


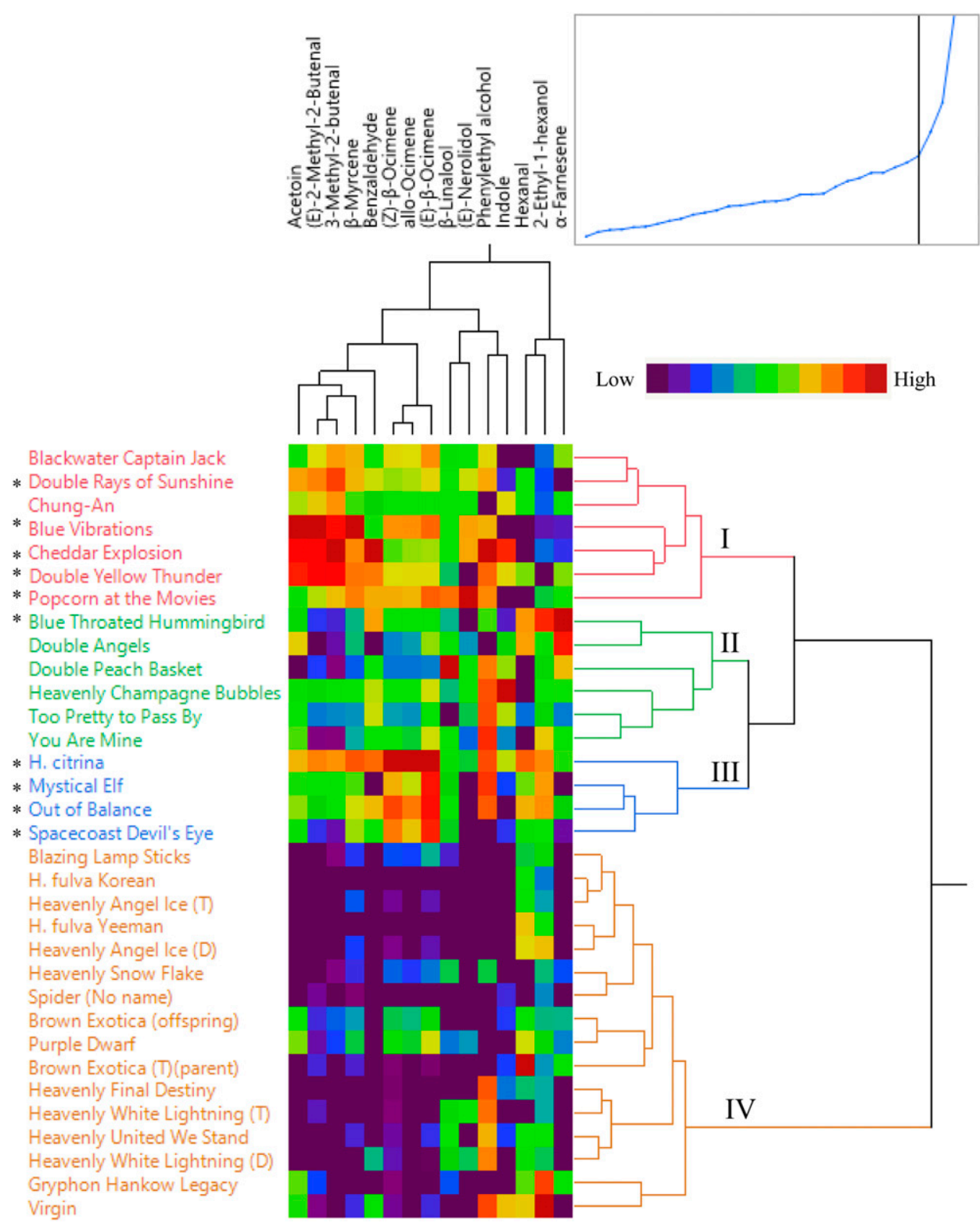

Fig. 6. Two-way cluster analysis of volatile compounds and Ohio daylilies $(\mathrm{n}=33)$. Daylilies clustered into four groups, labeled I to IV on the right side of the dendrogram. Daylilies that differentiated on the Ohio principal component analysis score plot are marked by an asterisk. Standardization of volatile content is by column mean and SD, with high values represented as red, average as green, and low as violet (spectral scale). (For interpretation of the references to color in this figure legend, the reader is referred to the online version of this article.)

this study. Of the volatiles listed in Lin et al. (2003), limonene (5989-27-5), 1-octen-3-ol (3391-86-4), benzeneacetaldehyde (122-78-1), 2-furanmethanol (98-00-0), cumyl alcohol (53660-7), cuminic aldehyde (122-03-2), hexanoic acid (142-62-1), benzenemethanol (100-51-6), and heptanoic acid (111-14-8) were not detected. Perhaps these compounds are unique to the aroma of the extracted essential oil of $H$. flava, or maybe these compounds were emitted at levels too low to detect with the system used in this study.

The highest percentage of fragrant genotypes, in terms of total volatile emissions, was found in Ohio, whereas the lowest percentage was found in Utah. These two areas differ substantially in temperature and relative humidity, which have been found to affect volatile emissions in some plant systems (Jakobsen and
Olsen, 1994; Vallat et al., 2005). These abiotic factors may have affected volatile emissions in this study, although more research is needed to determine how and to what extent these factors affect daylilies specifically. Aside from differences in climate, soil type, and other growth aspects, breeder goals and priorities also may be contributing factors to this aromatic differential. The Ohio collection was owned and managed by a largescale commercial breeder and germplasm collector interested in fragrance traits. Although not a priority, this hybridizer has occasionally selected for daylily cultivars with enhanced fragrance, which may partially explain the higher percentage of fragrant genotypes in this collection. Similarly, the lower aroma of the Utah daylilies could be explained in part by the priorities of the hobbyist breeder that owned and managed the collection. The intense heat and low humidity of the area poses significant challenges to daylily cultivation. Although daylilies are grown over a wide variety of conditions, they do not necessarily thrive in all of them (Gulia et al., 2009). Thus, this hobbyist breeder was focused on developing genotypes adapted to the Utah climate with desirable visual characteristics that do not fade under harsh sunlight, not fragrance.

Although the overwhelming majority of modern daylily breeding uses unregistered hybrids and cultivars, $H$. citrina and $H$. thunbergii could be valuable germplasm resources for the serious breeder interested in aroma traits. Despite their remarkably high fragrance, which is easily detected when one is in close proximity to them, the two species have rarely been used in modern daylily breeding. Indeed, a search of the Daylily Database (American Daylily Society, 2019) for daylilies bred from $H$. thunbergii yielded just 20 results of more than 90,000 registered cultivars. Only two of those hybrids were registered in the 2000s; the other 18 were bred between 1899 and 1949. H. citrina has been used a bit more often in modern breeding: a search of the database yielded 58 results, 44 of which have been registered since 2000 . The infrequent use of species in daylily breeding is likely due to interspecific cross-compatibility issues (Zhao et al., 2017). Such incompatibility barriers make it far more difficult, particularly for hobbyist breeders, to obtain viable seeds from a cross between species or between a species and cultivar; however, these barriers seem to be largely absent if two daylily 


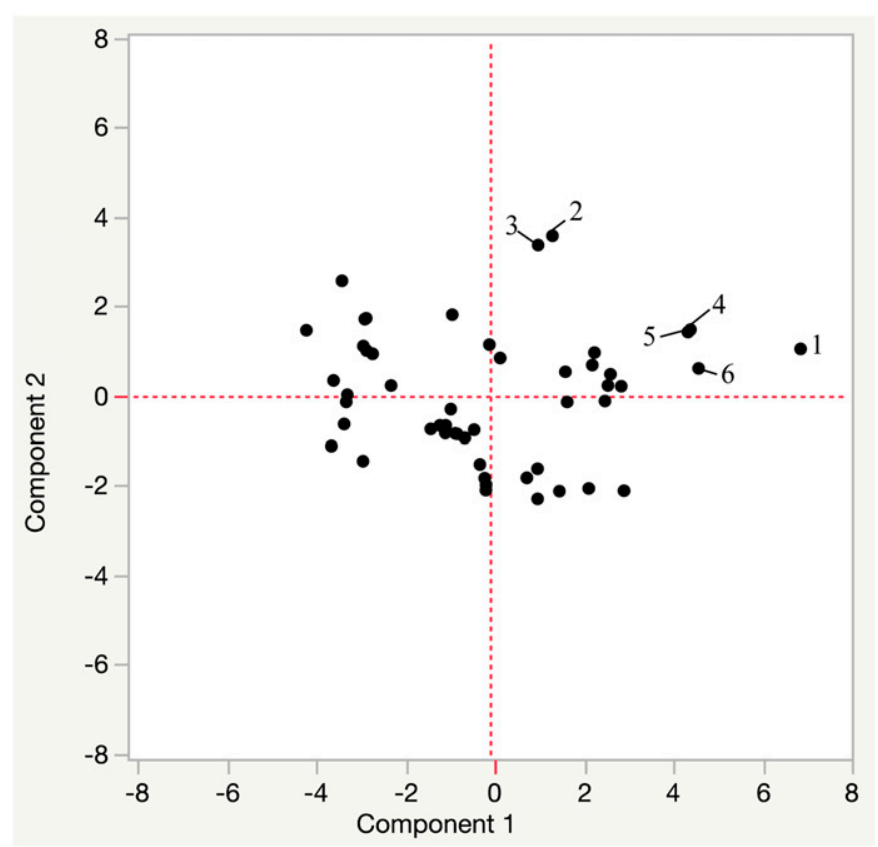

Fig. 7. Score plot of the first two principal components based on 15 volatile compounds detected in the floral scent of daylilies from Utah $(n=50)$. Components 1 and 2 explained $43.8 \%$ and $13.9 \%$, respectively, of the total observed variation. Daylilies that separated slightly from the large central cluster include (1) 'Cranberry Daiquiri', (2) 'Purple Fantasia', (3) 'Oh Great One', (4) 'Lover's Lemonade', (5) 'Samurai Jack', and (6) 'Glistening Accent'.

hybrids are crossed, as evidenced by the proliferation of registered cultivars that are bred from other hybrids. Although the species are certainly genetic resources of aroma compounds, it may be easier for some hybridizers to use highly scented hybrids, rather than the species, in a breeding program to enhance fragrance.

Of the entire study population of 147 daylilies, $80 \%(\mathrm{n}=$ 119 ) emitted less than half as much aroma as H. citrina. The low fragrance recorded for most of daylily hybrids in this study is similar to the results obtained by Jiao et al. (2016). In their evaluation of seven daylily species and 38 cultivars, only five species exhibited "intense or medium" floral fragrance, whereas the remaining daylilies were classified as having low or no floral scent. The stark contrast between the fragrance of the highly scented species and most hybrids illustrates the effect of hybridizers' traditional breeding objectives: the focus on flower form and coloration has indeed resulted in many daylilies with drastically reduced fragrance. However, not all hybrids sampled in this study had severely diminished fragrance: $17 \%(\mathrm{n}=26)$ emitted more than half as much volatiles as $H$. citrina, and of this group, seven were more than $70 \%$ as fragrant as $H$. citrina, including 'Blue Vibrations', 'Popcorn at the Movies', 'Out of Balance', and 'Spacecoast Blue Eyed Majesty'. The fragrance of these cultivars was very apparent and noticeable in the field. In contrast, none of the hybrids evaluated by Jiao et al. (2016) emitted high amounts of volatiles, with only four hybrids classified as having a "low scent." It is unclear how and why these hybrids are so fragrant. Perhaps they are more closely related to the highly scented species; however, it is difficult to determine the genetic background of many registered daylily cultivars. The best source of information on the genetic background of a registered cultivar is the Daylily Database maintained by the American Daylily
Society (2019). When a cultivar is registered, the hybridizer can provide its parentage...if it is known. Many cultivars lack parentage information entirely, whereas others are listed as a cross between two unnamed seedlings. Even if the parentage of a cultivar is provided, tracing that pedigree back often leads quickly to unknowns. For example, the parentage of the fragrant cultivar 'Out of Balance' can be traced back only two generations before some of the lines start hitting dead ends. More sophisticated molecular methods would be needed to explore the genetic background and relatedness of these cultivars. Regardless, the highly scented cultivars identified previously are genetic resources of aroma compounds, and they lack the major barriers to cross-compatibility exhibited by the species. Hybridizers could potentially use them in a breeding program to more easily develop unique, highly scented daylily cultivars that appeal to more consumers (Levin et al., 2012).

In the absence of meticulous pedigree records and sophisticated molecular techniques, some information about the relatedness of the daylily genotypes potentially could be gleaned from the HCA. HCA based on volatile content has been used to explore taxonomic relatedness in other plant systems, including citrus [Citrus sp. (Jing et al., 2015; Zhang et al., 2017)], parasitic plants [Orobanchaceae (Toth et al., 2016)], blueberries [Vaccinium sp. (Gilbert et al., 2015)], and Cymbopogon grasses (Khanuja et al., 2005); however, the results of clustering based solely on volatile profiles do not always coincide with established taxonomy. Inferring taxonomic relationships from the HCA of volatile profiles should be avoided unless these relationships can be confirmed by other approaches. But although HCA alone may not provide much information about the taxonomic relatedness of genotypes, it does provide information about their biochemical relatedness. In this study, HCA identified clusters of daylilies with exceptional fragrance profiles. Moreover, PCA and HCA identified some hybrids that, despite having low total emissions, emitted high amounts of individual volatiles. For example, 'Oh Great One' emitted more indole than any daylily evaluated. Indole is one of the most common nitrogen-containing compounds detected in floral scent, imparting floral characteristics at low concentrations, and fecal characteristics at high concentrations (Jabalpurwala et al., 2009; Knudsen et al., 2006). In addition, 'Bright Blaze of Magic' emitted the most acetoin and (E)-2methyl-2-butenal, whereas 'Wind Rider' emitted the most $(E)$ nerolidol and 2-ethyl-1-hexanol. These daylilies are genetic resources of specific volatiles and could serve as breeding material to introduce more diversity to daylily scent.

Of only 147 daylilies, this study identified hybrids that do have heightened aromas, some of which are nearly as fragrant as the species already. When standing near these species and hybrids, their fragrance is readily apparent and easily detected. Given the vast number of registered cultivars, many other fragrant hybrids likely exist. These results suggest that despite an overall lack of selection for aroma traits, fragrance has persisted in the germplasm, and a proportion of daylily hybrids seems to be naturally more fragrant. Furthermore, the limited artificial selection for fragrance by the breeder in Ohio has resulted in hybrids with increased scent. The elevated aroma of these daylilies pushes back on the notion that modern hybrids totally lack fragrance and suggests a greater deal of aromatic variation than initially thought. Hybridizers could use these already scented genotypes as starting material in a breeding program to increase fragrance even more. In a practical sense, 


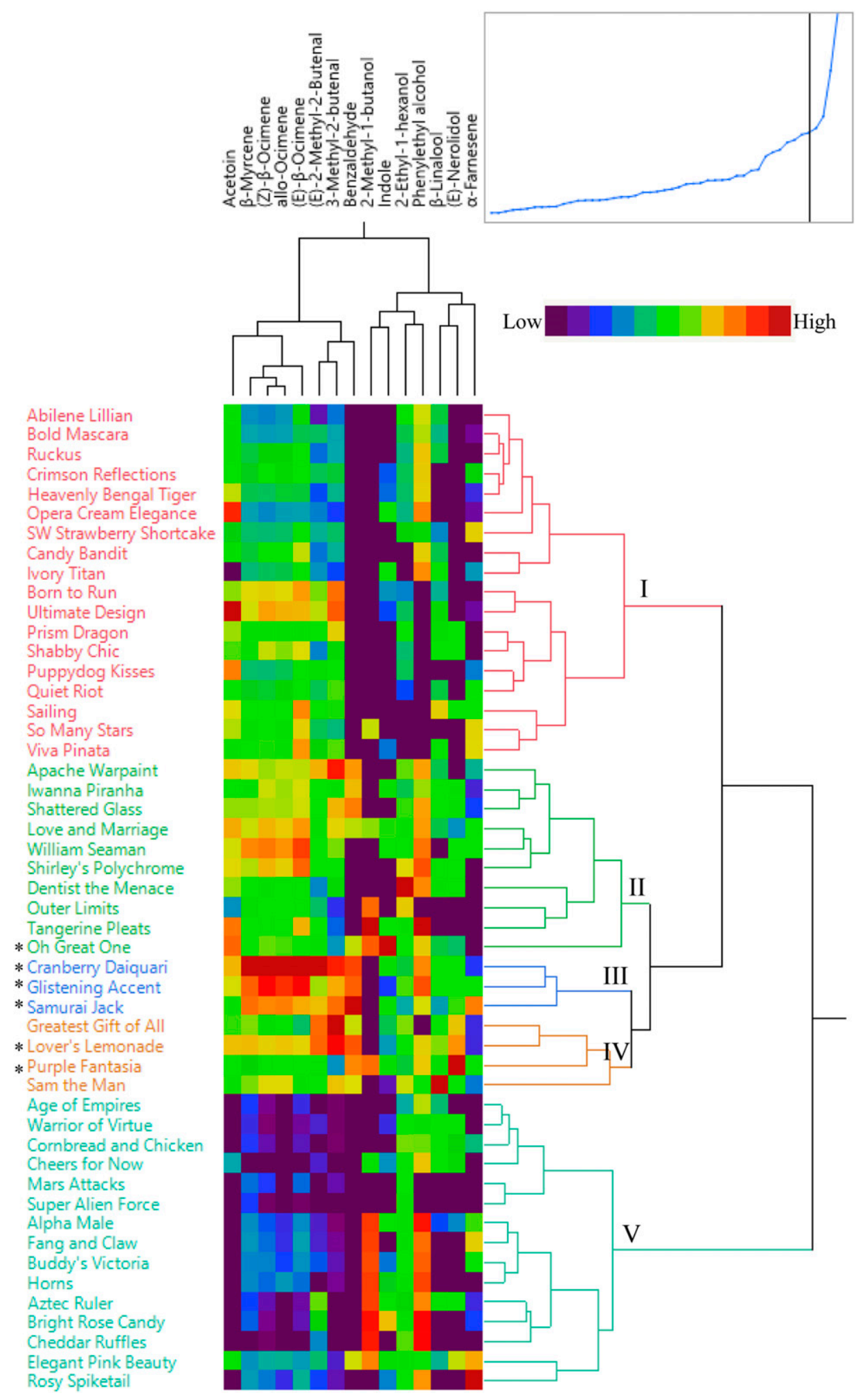

Fig. 8. Two-way cluster analysis of volatile compounds and Utah daylilies $(n=50)$. Daylilies clustered into five groups, labeled I to $\mathrm{V}$ on the right side of the dendrogram. Daylilies that differentiated on the Utah principal component analysis score plot are marked by an asterisk. Standardization of volatile content is by column mean and SD, with high values represented as red, average as green, and low as violet (spectral scale). (For interpretation of the references to color in this figure legend, the reader is referred to the online version of this article.)
Directions for future study are guided by the limitations of the present study. Because this research was conducted in the field on genotypes managed by different entities, there were several uncontrolled variables, including soil type and fertilization regimens, among others. No common genotype was evaluated in all locations. Evaluating the same genotype in all locations could have yielded useful information about environmental effects on volatile emissions, as well as the effects of different horticultural practices. As these daylilies were part of active breeding programs, destructive sampling methods were not used. Although the collection system was economical and practical for a field setting, it may not have been sensitive enough to detect volatiles at very low levels. Future work could involve more complete characterization of daylily fragrance using greenhouse-grown plants and a laboratory dynamic headspace collection system. Crosses could be made between scented genotypes based on their volatile profiles, and the volatile profiles of the offspring could be characterized and compared with that of the parents. Additional crosses would then be made after selecting for offspring with desirable volatile characteristics.

\section{Conclusions}

Using headspace volatile analysis and GC-MS, the volatile profiles of 147 Hemerocallis genotypes were investigated and 18 volatile compounds were identified. Five volatiles were reported in daylily aroma for the first time. This study demonstrated naturally occurring and artificially selected variation in the floral scent of three collections of Hemerocallis. Two species, $H$. citrina and $H$. thunbergii, were the most fragrant in terms of total volatile emissions. Although overall total emissions were low, and a high degree of similarity was seen in the fragrance profiles of most daylilies, PCA and HCA revealed a subset of daylily genotypes that differentiate from the rest. These genotypes hybridizers wishing to select for aroma traits do not need expensive analytical equipment to screen their populations for fragrance; all they need is a decent sense of smell. exhibited heightened overall aroma levels and/or heightened levels of specific volatiles, suggesting that despite a lack of selection for fragrance traits, fragrance persists in the daylily 
germplasm. These daylilies are genetic resources of volatile compounds, and hybridizers could use these, or noticeably scented daylilies from their own collections, in a breeding program to potentially create "novel," highly fragrant daylilies that stand out from the crowd of more than 90,000 existing cultivars and appeal to a broader base of consumers. Daylily hybridizers are an especially avid community of plant breeders who have wrought incredible transformations in the visual characteristics of daylilies. If they increase their focus on aromatic characteristics, they will undoubtedly transform the fragrance of daylilies, too.

\section{Literature Cited}

American Daylily Society. 2019. Daylily database. 9 Sept. 2019. $<$ https://www.daylilies.org/DaylilyDB/>.

Borda, A.M., D.G. Clark, D.J. Huber, B.A. Welt, and T.A. Nell. 2011. Effects of ethylene on volatile emission and fragrance in cut roses: The relationship between fragrance and vase life. Postharvest Biol. Technol. 59:245-252.

Dudareva, N., A. Klempien, J.K. Muhlemann, and I. Kaplan. 2013. Biosynthesis, function, and metabolic engineering of plant volatile organic compounds. New Phytol. 198:16-32.

Dudareva, N., F. Negre, D.A. Nagegowda, and I. Orlova. 2006. Plant volatiles: Recent advances and future perspectives. Crit. Rev. Plant Sci. 25:417-440.

Dudareva, N. and E. Pichersky. 2000. Biochemical and molecular genetic aspects of floral scents. Plant Physiol. 122:627-633.

Gilbert, J.L., M.J. Guthart, S.A. Gezan, M.P. de Carvalho, M.L. Schwieterman, T.A. Colquhoun, L.M. Bartoshuk, C.A. Sims, D.G. Clark, and J.W. Olmstead. 2015. Identifying breeding priorities for blueberry flavor using biochemical, sensory, and genotype by environment analyses. PLoS One 10:e138494.

Granato, D., J.S. Santos, G.B. Escher, B.L. Ferreira, and R.M. Maggio. 2017. Use of principal component analysis (PCA) and hierarchical cluster analysis (HCA) for multivariate association between bioactive compounds and functional properties in foods: A critical perspective. Trends Food Sci. Technol. 72:83-90.

Gulia, S.K., B.P. Singh, and J. Carter. 2009. Daylily: Botany, propagation, breeding. Hort. Rev. 35:193-220.

Huber, F.K., R. Kaiser, W. Sauter, and F.P. Schiestl. 2005. Floral scent emissions and pollinator attraction in two species of Gymnadenia (Orchidaceae). Oecologia 142:564-575.

Jabalpurwala, F.A., J.M. Smoot, and R.L. Rouseff. 2009. A comparison of citrus blossom volatiles. Phytochemistry 70:1428-1434.

Jakobsen, H.B. and C.E. Olsen. 1994. Influence of climatic factors on emission of flower volatiles in situ. Planta 192:365-371.

Jiao, F., Q. Liu, G.F. Sun, X.D. Li, and J.Z. Zhang. 2016. Floral fragrances of Hemerocallis L. (daylily) evaluated by headspace solid-phase microextraction with gas chromatography-mass spectrometry. J. Hort. Sci. Biotechnol. 91:573-581.

Jing, L., Z. Lei, G. Zhang, A.C. Pilon, D.V. Huhman, R. Xie, W. Xi, Z. Zhou, and L.W. Sumner. 2015. Metabolite profiles of essential oils in citrus peels and their taxonomic implications. Metabolomics 11:952963.

Kessler, D. and I.T. Baldwin. 2007. Making sense of nectar scents: The effects of nectar secondary metabolites on floral visitors of Nicotiana attenuata. Plant J. 49:840-854.

Khanuja, S.P.S., A.K. Shasany, A. Pawar, R.K. Lal, M.P. Darokar, A.A. Naqvi, S. Rajkumar, V. Sundaresan, N. Lal, and S. Kumar. 2005. Essential oil constituents and RAPD markers to establish species relationship in Cymbopogon Spreng. (Poaceae). Biochem. Syst. Ecol. 33:171-186.

Kishimoto, K., M. Nakayama, M. Yagi, T. Onozaki, and N. OyamaOkubo. 2011. Evaluation of wild Dianthus species as genetic resources for fragrant carnation breeding based on their floral scent composition. J. Jpn. Soc. Hort. Sci. 80:175-181.

Knudsen, J.T., R. Eriksson, J. Gershenzon, and B. Stahl. 2006. Diversity and distribution of floral scent. Bot. Rev. 72:1-120.

Levin, L.A., K.M. Langer, D.G. Clark, T.A. Colquhoun, J.L. Callaway, and H.R. Moskowitz. 2012. Using Mind Genomics ${ }^{\circledR}$ to identify the essential elements of a flower product. HortScience 47:1658-1665.

Lin, P., J. Cai, J. Li, W. Sang, and Q. Su. 2003. Constituents of the essential oil of Hemerocallis flava day lily. Flavour Fragrance J. 18:539-541.

Majetic, C.J., R.A. Raguso, S.J. Tonsor, and T. Ashman. 2007. Flower color-flower scent associations in polymorphic Hesperis matronalis (Brassicaceae). Phytochemistry 68:865-874.

Pichersky, E., J.P. Noel, and N. Dudareva. 2006. Biosynthesis of plant volatiles: Nature's diversity and ingenuity. Science 311:808-811.

Raguso, R.A., R.A. Levin, S.E. Foose, M.W. Holmberg, and L.A. McDade. 2003. Fragrance chemistry, nocturnal rhythms and pollination "syndromes" in Nicotiana. Phytochemistry 63:265-284.

Rodriguez-Enriquez, M.J. and R.T. Grant-Downton. 2013. A new day dawning: Hemerocallis (daylily) as a future model organism. AoB Plants 5:pls055.

Stewart-Jones, A. and G.M. Poppy. 2006. Comparison of glass vessels and plastic bags for enclosing living plant parts for headspace analysis. J. Chem. Ecol. 32:845-864.

Tholl, D., W. Boland, A. Hansel, F. Loreto, U.S.R. Rose, and J. Schnitzler. 2006. Practical approaches to plant volatile analysis. Plant J. 45:540-560.

Toth, P., A.K. Undas, F. Verstappen, and H. Bouwmeester. 2016. Floral volatiles in parasitic plants of the Orobanchaceae. Ecological and taxonomic implications. Front. Plant Sci. 7:312.

Vallat, A., H. Gu, and S. Dorn. 2005. How rainfall, relative humidity and temperature influence volatile emissions from apple trees in situ. Phytochemistry 66:1540-1550.

Widjaja, R., J.D. Craske, and M. Wootton. 1996. Comparative studies on volatile components of non-fragrant and fragrant rices. J. Sci. Food Agr. 70:151-161.

Zhang, H., Y. Xie, C. Liu, S. Chen, S. Hu, Z. Xie, X. Deng, and J. Xu. 2017. Comprehensive comparative analysis of volatile compounds in citrus fruits of different species. Food Chem. 230:316-326.

Zhao, J., L. Xue, X. Bi, and J. Lei. 2017. Compatibility of interspecific hybridization between Hemerocallis liloasphodelus and daylily cultivars. Scientia Hort. 220:267-274. 


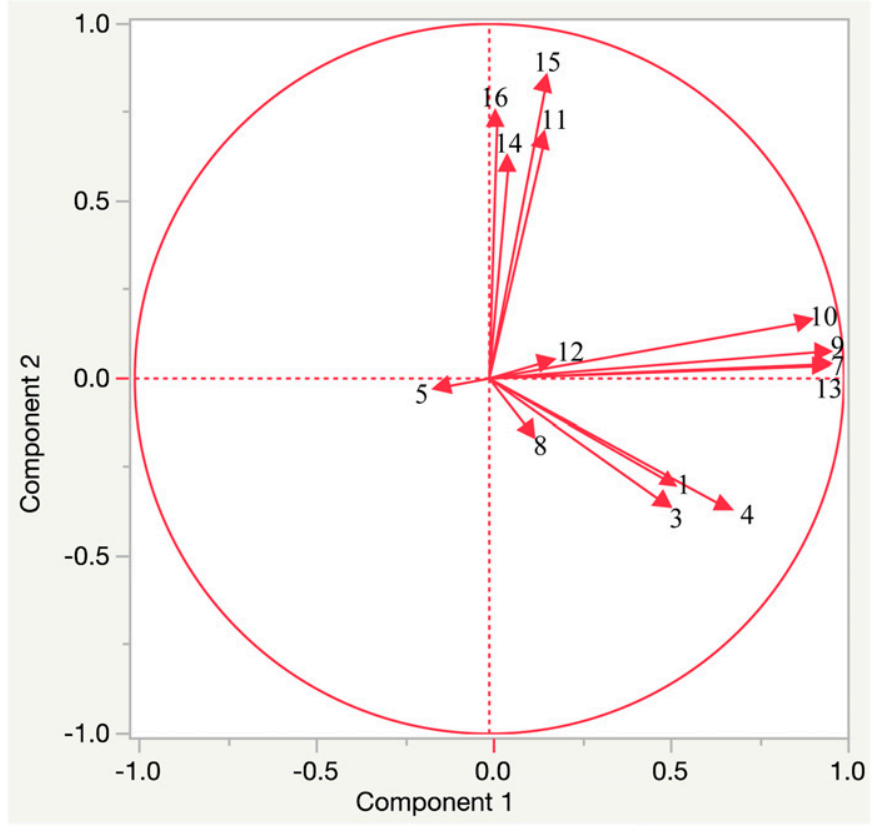

Supplemental Fig. 1. Loading plot of the volatile compounds used as variables in the principal component analysis of the Florida daylilies $(n=64)$. Components 1 and 2 explained $32.5 \%$ and $17.5 \%$, respectively, of the total observed variation. Refer to Supplemental Table 3 for key of volatile compounds and corresponding numbers.

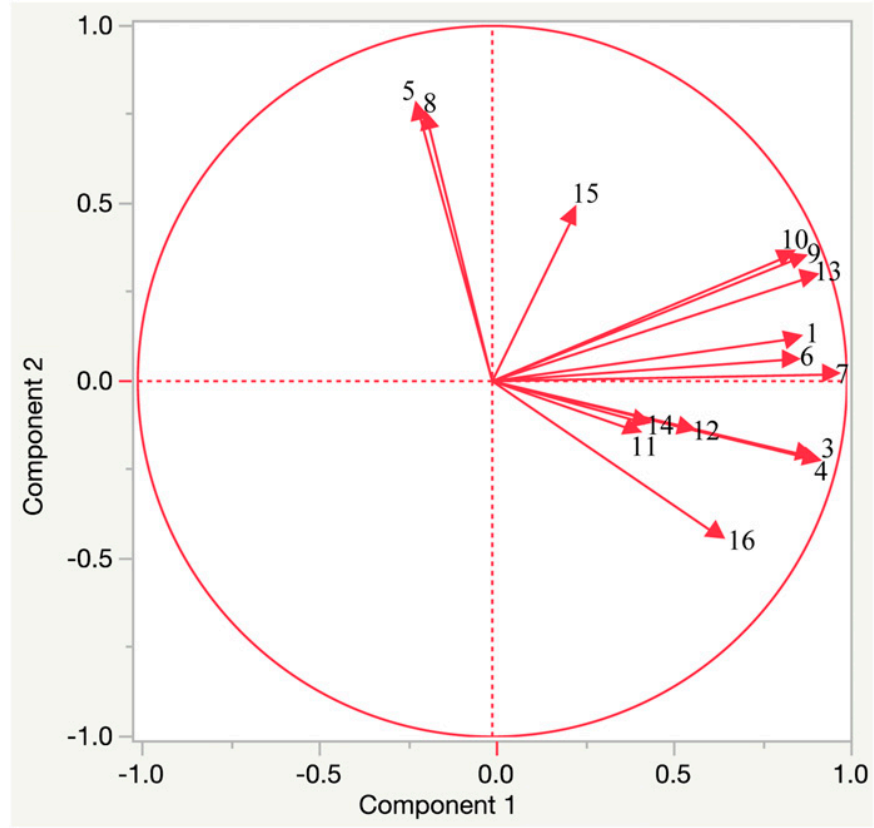

Supplemental Fig. 2. Loading plot of the volatile compounds used as variables in the principal component analysis of the Ohio daylilies $(\mathrm{n}=33)$. Components 1 and 2 explained $49.4 \%$ and $13.4 \%$, respectively, of the total observed variation. Refer to Supplemental Table 3 for key of volatile compounds and corresponding numbers.

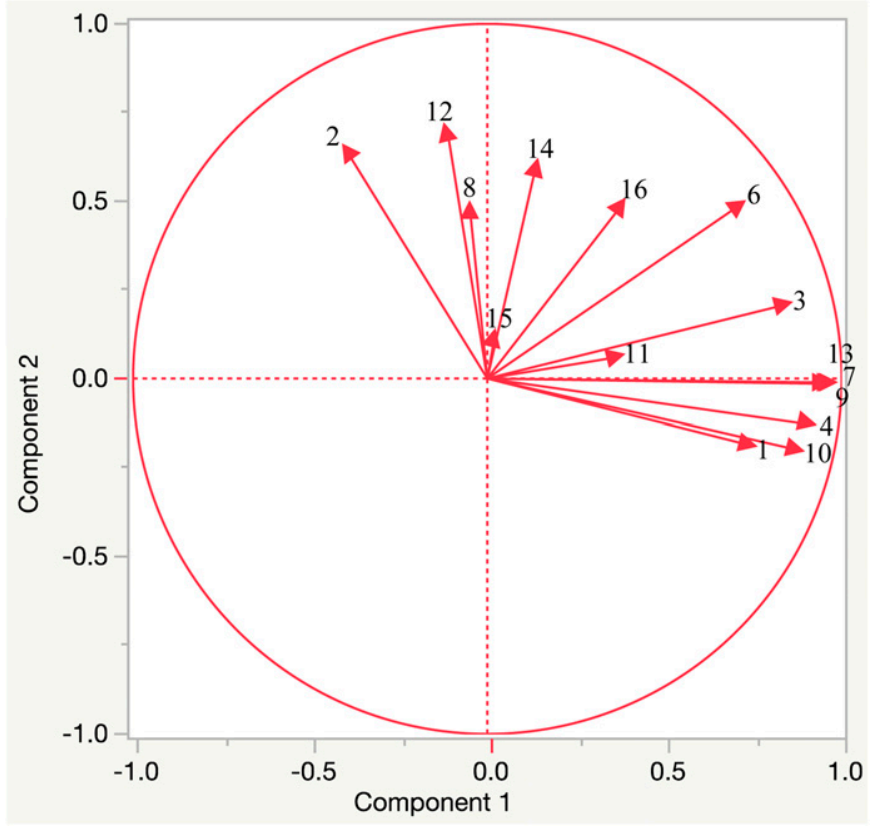

Supplemental Fig. 3. Loading plot of the volatile compounds used as variables in the principal component analysis of the Utah daylilies $(n=50)$. Components 1 and 2 explained $43.8 \%$ and $13.9 \%$, respectively, of the total observed variation. Refer to Supplemental Table 3 for key of volatile compounds and corresponding numbers. 
Supplemental Table 2. Loading coefficients for the first two principal components (PCs) of volatile compounds included as variables in the principal component analysis of daylilies in each study location. The coefficients indicate how much a compound affects a given PC: a compound that explains more variance is given a higher loading, whereas a compound that explains little to no variance has a loading value closer to zero.

\begin{tabular}{|c|c|c|c|c|c|c|}
\hline \multirow[b]{2}{*}{ Compound } & \multicolumn{2}{|c|}{ Florida } & \multicolumn{2}{|c|}{ Ohio } & \multicolumn{2}{|c|}{ Utah } \\
\hline & PC1 & PC2 & PC1 & PC2 & $\mathrm{PC} 1$ & $\mathrm{PC} 2$ \\
\hline Total variance explained & $32.5 \%$ & $17.5 \%$ & $49.4 \%$ & $13.4 \%$ & $43.8 \%$ & $13.9 \%$ \\
\hline Acetoin & 0.51 & -0.29 & 0.86 & 0.12 & 0.74 & -0.19 \\
\hline (E)-2-Methyl-2-butenal & 0.50 & -0.36 & 0.89 & -0.21 & 0.84 & 0.21 \\
\hline 3-Methyl-2-butenal & 0.66 & -0.36 & 0.90 & -0.22 & 0.90 & -0.13 \\
\hline Hexanal & -0.13 & -0.02 & -0.21 & 0.76 & & \\
\hline$\beta$-Myrcene & 0.95 & 0.04 & 0.96 & 0.02 & 0.96 & -0.01 \\
\hline 2-Ethyl-1-hexanol & 0.12 & -0.15 & -0.18 & 0.74 & -0.05 & 0.47 \\
\hline (Z)- $\beta$-Ocimene & 0.94 & 0.08 & 0.86 & 0.35 & 0.95 & -0.01 \\
\hline (E)- $\beta$-Ocimene & 0.90 & 0.16 & 0.83 & 0.35 & 0.88 & -0.20 \\
\hline$\beta$-Linalool & 0.16 & 0.67 & 0.40 & -0.14 & 0.37 & 0.06 \\
\hline Phenylethyl alcohol & 0.16 & 0.05 & 0.55 & -0.13 & -0.11 & 0.69 \\
\hline
\end{tabular}

Supplemental Table 3. Loading plot key for the numbered volatile compounds, included as variables in the principal component analysis of daylilies in each study location, corresponding to the loading plots shown in Supplemental Figs. 1 to 3.

\begin{tabular}{|c|c|}
\hline Compound name & $\begin{array}{c}\text { Corresponding } \\
\text { no. on loading plot }\end{array}$ \\
\hline Acetoin & 1 \\
\hline 2-Methyl-1-butanol & 2 \\
\hline (E)-2-Methyl-2-butenal & 3 \\
\hline 3-Methyl-2-butenal & 4 \\
\hline Hexanal & 5 \\
\hline Benzaldehyde & 6 \\
\hline$\beta$-Myrcene & 7 \\
\hline 2-Ethyl-1-hexanol & 8 \\
\hline$(Z)-\beta$-Ocimene & 9 \\
\hline (E)- $\beta$-Ocimene & 10 \\
\hline$\beta$-Linalool & 11 \\
\hline Phenylethyl alcohol & 12 \\
\hline allo-Ocimene & 13 \\
\hline Indole & 14 \\
\hline$\alpha$-Farnesene & 15 \\
\hline (E)-Nerolidol & 16 \\
\hline
\end{tabular}

